\title{
Effectiveness of Existing CAD-Based Research Work towards Screening Breast Cancer
}

\author{
Vidya Kattepura \\ Research Scholar \\ Siddhartha Academy of Higher Education \\ Tumkur, India
}

\author{
Dr. Kurian M Z \\ Registrar \\ Siddhartha Academy of Higher Education \\ Tumkur, India
}

\begin{abstract}
Accurate detection as well as classification of the breast cancer is still an unsolved question in the medical image processing techniques. We reviewed the existing Computer Aided Diagnosis (CAD)-based techniques to find that there has been enough work carried out towards both detection as well as classification of the breast cancer; however, all the existing techniques were implemented in highly controlled research environment. The prime contribution of this paper is it reviews some of the significant journals published during 2005-2016 and discusses its effectiveness thoroughly. The paper finally discusses about the open research issues that require a serious attention from the research community in order to address the existing issues. At the end, the paper makes some suggestion for carrying out future work direction in order to bridge the research gap explored from the existing system.
\end{abstract}

Keywords-Breast cancer detection; computer aided diagnosis; cancer; classification

\section{INTRODUCTION}

Breast cancer is one of the prominent reasons of mortality among the women in many countries. One of the most challenging and unfortunate part of this disease is that maximum women understand that they have breast cancer in latter stage and not in early stage. For this reason, preliminary prevention mechanism becomes one of the most challenging aspects [1]. The diagnosis of the cancer is carried out with respect to presence of micro-calcification and masses that are explored after the mammography is carried out. The x-ray based mammograms are considered as the prime screen test for evaluating as well as diagnosing the disease in its early stage, whereas its processing followed by analysis is considered to be best prognosis factor [2]. In a mammogram, the benign glandular tissues as well as the masses normally exist in very minimal contrast and are found to be quite blurred in nature. This strikes a significant challenge to the radiologist to understand the significance of the disease. Therefore, various professionals and researchers have been evolving up with a scheme that can assists in proper interpretation and diagnosis. Till date, there is no such mechanism for resisting the breast cancer to occur. Hence, the preliminary detection is the first significant step towards the diagnosis and treatment of the breast cancer. In this regards, Computer Aided Diagnosis (CAD) based techniques assists in providing us various mechanism for both prognosis as well as diagnosis [3], [4]. From this viewpoint, mammography is one of the most practiced methods for early screen of breast cancer. It has also the added advantage of cost effectiveness and higher availability. Irrespective of its advantages, mammography suffers from the issues of low reliability when it comes to detection of dense breast tissues. This causes higher sensitivity to the dense tissue but better detection performance over the micro calcification [5], [6]. There are different forms of abnormal conditions found in mammograms e.g. architectural distortion, microcalcification, asymmetry, masses, etc. Because of this reason, maximum quantity of the mammograms will be required to be cross checked by the radiologists who are less in numbers in comparison to mammograms. This causes significant misdiagnosis as one radiologist has to interpret many numbers of mammograms by their naked eyes. This process eventually causes stress to eyes leading to error in interpretation. Adoption of digital image processing as well as pattern recognition in $\mathrm{CAD}$ techniques is quite frequent in order to perform an effective detection followed by classification of the breast cancer. Unfortunately, the most frequently used screening programs of breast cancer cannot forecast the prognosis for the situation of the patient is really suffering from breast cancer. In such condition, the cells spread very fast and take the shape of malignancy stage which is less likely to be controlled by common drugs. Apart from this problem, another bigger set of problem is medical image itself is a quite challenging signal which is very different from natural images. This is also the prime reason why normal object-based detection or classification approaches are not directly applicable on medical images. Normally, medical images are bigger in size and they are usually grey scale, which makes the diagnosis more difficult. Poor illumination condition, artifacts, external factors while taking the images are also equally responsible for increasing challenges in diagnosis of medical images.

Apart from the discussion presented in [7]-[9], there are no review work till date to evaluate the effectiveness of existing techniques of breast cancer. Hence, this paper presents an updated review of effectiveness in CAD-based method for screening breast cancer with respect to detection mechanism and classification techniques. Section II discusses about the essential fundamental information about the CAD-based screening of breast cancer. Section III discusses about the existing research contribution towards detection and classification followed by briefing of open research issues in Section IV. Finally, Section V concludes the paper. 


\section{PREAMBLE: BREAST CANCER SCREENING}

Computer Aided Diagnosis (CAD) is the most frequently used practices for screening the breast cancer. The primary target of using CAD schemes is mainly to segregate the region containing masses that is suspected with carcinoma from parenchyma in the background [10]. Usage of CAD schemes allows the radiological images of breast cancer in the form of numerous regions that doesn't intersect with each other followed by extracting the segment within an image bearing strong clinical significance (see Fig. 1).
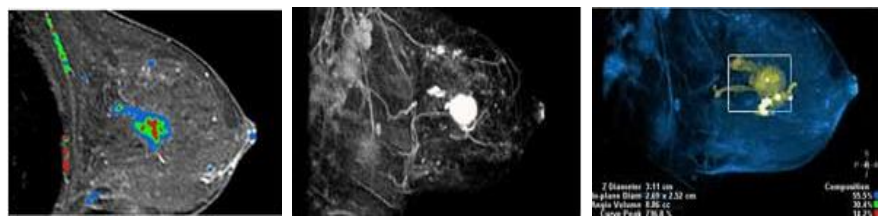

Fig. 1. CAD-generated images of breast cancer.

The CAD mechanism has been used in the medical sector in order to enhance the level of efficiency as well as accuracy from the radiological images so that physician can take decision of treatment based on the criticality of the disease from the $\mathrm{CAD}$ generated images. All the radiological images are interpreted by the CAD system which is again divided into two types, i.e. 1) CADe; and 2) CADx [11]. CADe is basically used for detection of the region of the breast inflicted with cancer while CADx is used for making essential classification. Hence, both the techniques are quite important for making decisions. At present, there are various other techniques based on image processing e.g. discrete wavelet transform [12], fractal analysis [13], Markov random field [14], etc. Although detection becomes very important when it comes in early stages, however, owing to the complex nature of the disease, it is quite difficult to perform positive detection. The frequently exercised clinical belief of positive detection of breast cancer is the presence of masses or micro-calcified part. This is the prime reason of maximum work towards cancer detection is based on microcalcification and detection of masses [15]-[17].

\section{A. Tools for Diagnosis}

Basically, the mechanism of screening breast cancer is by using mammogram, magnetic resonance imaging (MRI), as well as clinical breast examination. The clinical breast examination is carried out by visually examination while mammogram is carried out using $\mathrm{x}$-ray to reconstruct breast image. Breast MRI uses magnetic fields and is highly invasive as compared to mammography (see, Fig. 2).



(a) Mammogram

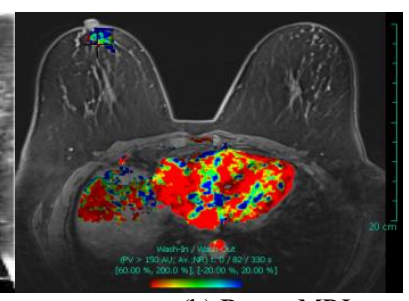

(b) Breast MRI
Fig. 2. Screening mechanism of breast cancer.

\section{B. Problems in Existing CAD System}

The common imaging tools to extract images are ultrasound, mammography, positron emission mammography, molecular breast imaging, etc. These techniques are frequently used for identification of cancer from dense tissue within a breast. Although CAD based system to generate radiological image of breast give better visual field for assessing the presence of cancer, but yet physician believe that they can be wrong too. For an example, mammogram is one of the effective mechanism to find cancer but only in early stage however, accuracy of mammogram is still questionable as it is still less invasive. Basically, a mammogram can never provide that the abnormally identified area is infected with cancer. It is only based on the skill of radiologist or the physician that the true inference could come. This is the reason certain physician suggest going for high end screen test for breast cancer. Another bigger limitation of mammogram is that it fails its diagnostic capability for dense tissue within a breast. At the same time, when people believe that breast MRI is highly accurate and suitable for cancer detection, radiologist believes it in otherwise. It is because breast MRI uses a contrast agent causing reaction [18] and occurrences of false positives are more in breast MRI as compared to mammogram. There are certain types of biopsies that are carried out on the basis of breast MRI. It is only possible in breast MRI and not in mammogram, but it has dependency of highly skilled professional to do so making it one of the expensive diagnostic processes compared to mammograms. Frequent occurrences of the higher false positives also provoke the doctor to suggest for extra biopsies causing anxiety and stress to the patients. Ultrasound is another frequently used practice to differentiate between solid masses and cyst. Although, sometime it could be also employed to differentiate between malignant and benign stage, but still usage of ultrasound is not much recommended as compared to mammogram in breast cancer.

Moreover, risks of false positives are more in CAD-based techniques. Certain area can be interpreted as abnormal in mammogram but in originality, it may be quite normal, hence, it gives rise to false positives. There is another type of cancer called as ductal carcinoma in situ which is not at all a type of invasive cancer. It may be rather called as benign and never malignant as it just stay within the breast duct and never spreads out. This is the condition when there is a need of an effective classification process of detecting it as benign or malignant type of cancer. Hence, an effective algorithm is required to solve such issues.

Normally, a CAD-based algorithm [19], [20] needs its input in the form of digitized image. Hence, if the image is generated by any other analog means than it has to be altered to digital signal for applying CAD based algorithms before applying the detection or classification approaches. At present, majority of the methods of validating the CAD system is basically based on laboratory-based experiments and no such computational modeling is yet prototyped for this cause. However, sensitivity and specificity is the only mechanism to check the legitimacy of the claims of absence or presence of cancer for a given image. The next section will brief about the existing research work towards the detection and classification of the breast cancer. 


\section{EXISTING RESEARCH WORK}

This section discusses about the existing research work being carried out towards measuring effectiveness of imaging technologies evolved for detection and classification techniques of breast cancer.

\section{A. Detection Techniques}

Majority of the detection techniques adopts CAD techniques for assessing the criticality of the radiological image of breast. The prime purpose is to ensure that the image provides more information that could assist in detection of the critical regions infected with carcinoma. One of the recent studies shows that human factor significant affects in the cancer detection process. The study on such direction was carried out by Singh et al. [21] by introducing descriptor-based technique called as polar complex exponential transform followed by feature extraction of region suspected with cancer. The study outcome was testified with ROC curve to find more than $97 \%$ of accuracy. Usage of imaging from Ultra-Wide Band microwave was considered in the study of Li et al. [22] who have implemented a detection technique on the basis of empirical mode decomposition. The author have used experimental approach using oscilloscope, switching matrix, amplifiers, differentiator, and pulse pattern generator for performing detection of cancerous part of breast. Adoption of microwave system as well as similar experimental approach was also found in the work carried out by Santorelli et al. [23]. A circuit board of multiple layers is designed in order to monitor breast cancer. Thermo Acoustic Imaging is another novel form of medical imaging techniques that was also reported to be used for breast cancer detection. A study conducted by Wang et al. [24] has used contrast while performing Thermo Acoustic Imaging. An experimental as well as simulation-based approach was used considering realtime breast phantoms. Yin et al. [25] have adopted ultra-wide band imaging mechanism to perform detection of breast cancer. The authors have focused on tumor detection problem due to certain complex tissues and uses correlation-based pairwise weighting as a solution. The study outcome was found to be resilient against artifacts and exhibits enhanced detectability skills. Song et al. [26] has adopted experimental approach to solve the problem of breast cancer detection. The authors have used circuit design using Gaussian monocycle pulse with antenna array of ultra-wide band. The technique allows the generated signal to be transmitted on specific frequency to transmitter using switching matrix. A prototype is designed for identification of breast cancer with 1-cm accuracy limit. Kwon et al. [27] have adopted time domain for enhancing the radiological image generated by microwave imaging system in order to perform detection of cancer. The technique has used Gaussian band pass filtering using simulation-based approach. The study outcome was evaluated with respect to signal-to-mean ratio. Adoption of medical images from ultra-wide band was also seen in the work carried out by Jalilvand et al. [28] where the authors have used a threedimensional identification system for breast cancer using bowtie antenna. Basically, authors have implemented nearfield imaging mechanism. Kirshin et al. [29] have presented a study where medical image of breast cancer was obtained from both microwave radar and thermo acoustic imaging for enhanced detectability. The study uses time-domain approach of finite difference. The study outcome was studied with respect to Peak-To-Side Lobe Ratio (PSLR) and false alarms. Shahzad et al. [30] have presented an enhanced beam formingbased technique in order to mitigate the phase effects as well as dispersion using a novel pre-filtering approach. Hossain et al. [31] have presented a technique using enhancement for microwave imaging that could leverage the detection system significantly. Using MRI image phantoms, the technique applies beamspace transformation in order to mitigate the skinbased artifacts and enhance the imaging performance. Bassi et al. [32] have presented an experimental approach where CMOS is used along with antenna patch for obtained microwave images of breast cancer. The unique radio transceiver has been designed for this purpose in order to identify tumor on breast phantom. Peter et al. [33] have developed a unique imaging mechanism using single frequency in order to enhance the performance of breast cancer detection. The study outcome was testified using ROC curve. Ruvio et al. [34] have presented a discussion to show that multi-frequency-based technique with numerous classifications of signals provides an effective detection performance. Guardiola et al. [35] have presented a imaging technique by integrating tomography with three dimensional microwave for the similar purpose of breast cancer detection. However, the focus is more on algorithm efficiency than on imaging. Using three dimensional phantoms, the microwave signals are found to provide better detection capabilities. Similar category of the work is also carried out by Grzegorczyk et al. [36]. Usage of thermoacoustic imaging along with microwaves has been used for generating reconstructed image for breast cancer detection by Wang et al. [37]. Aguilli et al. [38] have discussed about a Matlab-based approach for converting MRI image in the form of contrast maps in order to assists in detection of breast cancer.

\section{B. Classification Techniquess}

This technique is more towards understanding the type of the disease or the specification of the criticality. Majority of the research technique evolved in due course of time are related to categorizing the cancer stages and disease criticality based on the successful detection techniques. Hence, the successful classification techniques can only happen if the detection technique is successful. Work in this direction has been recently carried out by Bekker et al. [39] where a classifier has been designed using logistic-based technique as well as stochastic approach. The prime idea was to classify benign and malignant stage of cancer. The study outcome evaluated with respect to performance parameters e.g. accuracy, sensitivity, specificity, etc. A problem of classifying micro-calcification was also investigated by Chen et al. [40] where multi-scale morphology is used for analyzing the topology of the microcalcification. The system also generates graph of microcalcification depending on the spatial connectivity. Gangeh et al. [41] have presented a technique of computer-aided theragnosis of non-invasive nature. The technique has adopted an attribute based on the kernel using learning depending on arbitrary under sampling. Spanhol et al. [42] introduces a technique where the comprehensive dataset was developed in order to perform analysis / classification of breast cancer. The outcome was found with $85 \%$ of accuracy. Vu et al. [43] have presented a technique of involuntary feature extraction using 
dictionary learning technique. It was found that the dictionary significantly assists in classification process. Uniyal et al. [44] have implemented a technique that uses time-series analysis to perform classification of the malignancy in breast cancer. The technique generates a map of malignancy using machine learning approach. Soares et al. [45] have developed a modeling technique that performs classification of significant features using multifractal scaling exponent. Krawczyk et al. [46] have presented a technique of classification based on thermogram image of breast. The technique extracts thermogram images for analyzing bilateral symmetries and applies ensemble classification technique. The technique develops classifier based on neural network and evolutionary algorithm. Liu et al. [47] have developed a new feature extraction technique using supervised learning technique over mammogram. An extensive statistical procedure was adopted to testify the effectiveness of the proposed study. Amaral et al. [48] have presented a technique that performs involuntary classification and ranking of the significant portions in microarrays. Ashraf et al. [49] have introduced a technique of classification of breast cancer using Markov Random Fields. The technique is based on single channel Markov Random Fields. Filipczuk et al. [50] have presented a technique of diagnosing breast cancer using cytological images. The study outcome was found to possess $98 \%$ of effectiveness to furnish potential information. Tripoliti et al. [51] have presented a classification technique using random forest. The technique also utilizes fitting method in order to obtain better accuracy. Dundar et al. [52] have introduced ROI-based classification technique along with training-based approach for categorizing intraductal lesion in breast. The author uses a prototyping along with feature extraction and training of classifier in order to achieve a better classification outcome. The study outcome was evaluated with respect to sensitivity and specificity. ROI-based technique was also adopted by Fraschini et al. [53] where a wavelet as well as neural network was used for incorporating the classification process of breast cancer. Usage of metrological characteristics was seen in the work carried out by Mencattini et al. [54] in order to perform validation of the extracted features as well as its selection process. The technique also uses Monte Carlo curve in order to carry out simulation. Using ROI-based approach, the main classification algorithm of the presented technique will perform extraction of section with clinical interest that consists of segmentation, feature extraction, feature selection, and classification of tumoral mass. Classification technique was also applied on the medical images captured using ultra-wide band signals. One of such study was carried out by Teo et al. [55].

TABLE I. SUMMARY OF EXISTING RESEARCH IN BREAST CANCER DETECTION

\begin{tabular}{|c|c|c|c|}
\hline Author & Problem & Technique Applied & Remarks \\
\hline Singh et al. [21] & $\begin{array}{l}\text { Minimizing human-factor involvement } \\
\text { in detection }\end{array}$ & $\begin{array}{l}\text { Polar Complex Exponential Transform, } \\
\text { Wavelets, Neural Network }\end{array}$ & $\begin{array}{l}\text { Pros: } 97 \% \text { accuracy better than } 7 \text { existing } \\
\text { techniques } \\
\text { Cons: Accuracy depends on training. }\end{array}$ \\
\hline Li et al. [22] & $\begin{array}{l}\text { Tumor detection from reconstructed } \\
\text { image }\end{array}$ & Experimental, 3D printing & $\begin{array}{l}\text { Pros: Less accuracy for detecting position } \\
\text { of cancer } \\
\text { Cons: Not Scalable, will not support faster } \\
\text { response }\end{array}$ \\
\hline Santorelli et al. [23] & $\begin{array}{l}\text { Imaging system for Tumor detection } \\
\text { from reconstructed image }\end{array}$ & Experimental, microwave circuits, & $\begin{array}{l}\text { Pros: Less accuracy for detecting position } \\
\text { of cancer } \\
\text { Cons: Not Scalable, will not support faster } \\
\text { response }\end{array}$ \\
\hline $\begin{array}{l}\text { Wang et al. [24], Wang et } \\
\text { al. [37]. }\end{array}$ & Thermo Acoustic Imaging & Experimental, Simulation & $\begin{array}{l}\text { Pros: Could use thermo-acoustic imaging } \\
\text { Cons: Doesn't support faster response, no } \\
\text { comparative analysis }\end{array}$ \\
\hline Yin et al. [25] & $\begin{array}{l}\text { Tumor detection from reconstructed } \\
\text { image }\end{array}$ & Correlation-based pairwise weighting & $\begin{array}{l}\text { Pros: Resistive of artifacts. } \\
\text { Cons: No discussion of accuracy }\end{array}$ \\
\hline Song et al. [26] & Imaging system for Cancer detection & $\begin{array}{l}\text { Experimental, CMOS circuits, microwave } \\
\text { imaging }\end{array}$ & $\begin{array}{l}\text { Pros: Good Detection performance } \\
\text { Cons: No comparative analysis }\end{array}$ \\
\hline Kwon et al. [27] & Enhancing image for cancer detection & Simulation, Gaussian band pass filtering & $\begin{array}{l}\text { Pros: -NIL- } \\
\text { Cons: Doesn't show better outcomes, } \\
\text { needs more tuning. }\end{array}$ \\
\hline Jalilvand et al. [28] & Imaging system for Detection of Tumor & $\begin{array}{l}\text { Experimental, three-dimensional imaging, } \\
\text { bowtie antenna }\end{array}$ & $\begin{array}{l}\text { Pros: Good demonstration of detection on } \\
\text { breast phantoms } \\
\text { Cons: No comparative analysis }\end{array}$ \\
\hline Kirshin et al. [29] & Imaging system for Cancer detection & $\begin{array}{l}\text { Microwave radar and thermo acoustic } \\
\text { image }\end{array}$ & $\begin{array}{l}\text { Pros: Good PSLR performance } \\
\text { Cons: No comparative analysis }\end{array}$ \\
\hline Shahzad et al. [30] & $\begin{array}{l}\text { Imaging system for Cancer detection in } \\
\text { early state }\end{array}$ & Beamforming, prefiltering & $\begin{array}{l}\text { Pros: Good accuracy } \\
\text { Cons: No comparative analysis }\end{array}$ \\
\hline Hossain et al. [31] & Imaging system for Cancer detection & Time-Reversal, Beamspace transformation & $\begin{array}{l}\text { Pros: Good SNR performance } \\
\text { Cons: Leads to Computational } \\
\text { Complexity for big dataset }\end{array}$ \\
\hline Bassi et al. [32] & Imaging system for Cancer detection & $\begin{array}{l}\text { Prototyping transceiver using CMOS, } \\
\text { microwave image, }\end{array}$ & $\begin{array}{l}\text { Pros: good performance } \\
\text { Cons: No comparative analysis }\end{array}$ \\
\hline Peter et al. [33] & Imaging system for Cancer detection & Terahertz frequency, & $\begin{array}{l}\text { Pros: } 70 \% \text { lesser discrimination value } \\
\text { Cons: No comparative analysis }\end{array}$ \\
\hline Ruvio et al. [34] & Comparative analysis of imaging system & $\begin{array}{l}\text { Multifrequency Multiple Signal } \\
\text { Classification }\end{array}$ & $\begin{array}{l}\text { Pros: good detection capability } \\
\text { Cons: No comparative analysis }\end{array}$ \\
\hline $\begin{array}{l}\text { Guardiola et al. [35], } \\
\text { Grzegorczyk et al. [36] }\end{array}$ & Imaging system for Cancer detection & Tomography, microwaves, & $\begin{array}{l}\text { Pros: Supports complex images of breast } \\
\text { cancer } \\
\text { Cons: No comparative analysis }\end{array}$ \\
\hline
\end{tabular}


TABLE II. SUMMARY OF EXISTING RESEARCH IN BREAST CANCER ClasSIFICATION

\begin{tabular}{|c|c|c|c|}
\hline Author & Problem & Technique Applied & Remarks \\
\hline Bekker et al. [39] & $\begin{array}{l}\text { Classification of micro- } \\
\text { calcification }\end{array}$ & Logistic regression, stochastic & $\begin{array}{l}\text { Pros: Simple empirical model } \\
\text { Cons: Lesser Accuracy }\end{array}$ \\
\hline Chen et al. [40] & $\begin{array}{l}\text { Classification of micro- } \\
\text { calcification }\end{array}$ & Multi-scale morphology, topology & $\begin{array}{l}\text { Pros: } 96 \% \text { of accuracy } \\
\text { Cons: Doesn't emphasize on computational } \\
\text { complexity. }\end{array}$ \\
\hline Gangeh et al. [41] & Early cancer detection & Machine learning, multi-parametric approach & $\begin{array}{l}\text { Pros: } 90 \% \text { accuracy } \\
\text { Cons: No comparative analysis }\end{array}$ \\
\hline Spanhol et al. [42] & Classification of breast cancer & Introduced a new dataset & $\begin{array}{l}\text { Pros: Simpler Technique } \\
\text { Cons: Accuracy controlled by training }\end{array}$ \\
\hline Vu et al. [43] & Classification problems & Dictionary-based learning & $\begin{array}{l}\text { Pros: } 97 \% \text { of classification accuracy } \\
\text { Cons: Doesn't emphasize on computational } \\
\text { complexity }\end{array}$ \\
\hline Uniyal et al. [44] & Classification of malignancy & Random forest, time-series, machine learning & $\begin{array}{l}\text { Pros: Simple ROI based classification } \\
\text { Cons: No Effective comparative analysis }\end{array}$ \\
\hline Soares et al. [45] & Diagnosis of breast cancer & Multifractal image analysis & $\begin{array}{l}\text { Pros: Good Classification } \\
\text { Cons: No Effective comparative analysis }\end{array}$ \\
\hline $\begin{array}{l}\text { Krawczyk et al. } \\
{[46]}\end{array}$ & $\begin{array}{l}\text { Classification from thermogram } \\
\text { image }\end{array}$ & $\begin{array}{l}\text { Bilateral symmetry analysis, neural network } \\
\text { evolutionary algorithm }\end{array}$ & $\begin{array}{l}\text { Pros: Good Accuracy } \\
\text { Cons: Not computationally efficient technique }\end{array}$ \\
\hline Liu et al. [47] & $\begin{array}{l}\text { Feature selection for } \\
\text { classification }\end{array}$ & Supervised learning techniques & $\begin{array}{l}\text { Pros: Simple approach. } \\
\text { Cons: Only comparable with learning-based } \\
\text { techniques }\end{array}$ \\
\hline Amaral et al. [48] & $\begin{array}{l}\text { Classification based on } \\
\text { microarray }\end{array}$ & Bag of visual words & $\begin{array}{l}\text { Pros: Good Accuracy } \\
\text { Cons: No Effective comparative analysis }\end{array}$ \\
\hline Ashraf et al. [49] & Classification problems & Markov Random Fields, supervised training & $\begin{array}{l}\text { Pros: Higher true positive cases } \\
\text { Cons: Only comparable with learning-based } \\
\text { techniques }\end{array}$ \\
\hline $\begin{array}{l}\text { Filipczuk et al. } \\
\text { [50] }\end{array}$ & $\begin{array}{l}\text { Diagnosis from cytological } \\
\text { images }\end{array}$ & Hough transform, support vector machine & $\begin{array}{l}\text { Pros:98\% effectiveness } \\
\text { Cons: Applicable to only one type of image } \\
\text { database, }\end{array}$ \\
\hline Tripoliti et al. [51] & Classification & Random forest & $\begin{array}{l}\text { Pros: Good Accuracy } \\
\text { Cons: No Comparative Analysis }\end{array}$ \\
\hline Dundar et al. [52] & Classification & Feature extraction, training & $\begin{array}{l}\text { Pros: } 84 \% \text { of accuracy } \\
\text { Cons: Computationally expensive process }\end{array}$ \\
\hline $\begin{array}{l}\text { Fraschini et al. } \\
\text { [53] }\end{array}$ & Classification & Wavelet, neural network & $\begin{array}{l}\text { Pros: Good Accuracy } \\
\text { Cons: No Comparative Analysis }\end{array}$ \\
\hline $\begin{array}{l}\text { Mencattini et al. } \\
{[54]}\end{array}$ & Classification & ROI, Feature extraction, Segmentation, Selection & $\begin{array}{l}\text { Pros: Good technique to differentiate. } \\
\text { Cons: No Comparative Analysis }\end{array}$ \\
\hline Teo et al. [55] & Classification from UWB signal & Correlation & $\begin{array}{l}\text { Pros: Can identify multiple morphology of } \\
\text { lesion. } \\
\text { Cons: No Comparative Analysis }\end{array}$ \\
\hline
\end{tabular}

IV. OPEN RESEARCH ISSUES

From the previous section, it was found that the existing research techniques have presented some of the deliberate attempts towards detection as well as classification of the breast cancer. The scopes as well as the advantages of the existing techniques are summarized in Tables 1 and 2. This section will explicitly discuss about the open research issues after reviewing the existing techniques of detection and classification methods:

- More Emphasis on Signal Generation Process: It is widely seen that majority of the detection scheme have 
emphasized on its microwave technologies and its generated medical images. The positive point in this is all such work has been carried out using hardwarebased approach to prove its efficiencies of detection using breast phantoms. However, the major limitation will be the lesser extent of benchmarking and less effective comparative analysis. Another open research issues is that even using such sophisticated devices, the rate of false positives are not diminished to a significant extent. Because of this phenomenon, usage of ultrawide band microwave images are more as compared frequently used MRI images in existing detection techniques.

- Less Emphasis on Feature Extraction Process: Although many of the existing techniques have extracted features but it has not been emphasized from mathematical viewpoint. It's the simple low-level feature that has been extracted. It is widely known that transform-based techniques are good for feature extraction but its usage was found quite limited in case of both detection and classification of breast cancer for existing system. Moreover existing feature selection process are less optimized and more iterative causing low impact on increasing accuracy of the detection process.

- Less Optimized Frameworks: Usage of computational framework is quite less when it comes to classification process of breast cancer. Some of the simplified works on micro-calcification gives a good shape of modeling but they cannot be re-used under different circumstances e.g. change of dataset, or inclusion of new algorithms, etc. Moreover, the ROC curve, accuracy, specificity, sensitivity, etc. are not found to achieve a significant change in their patterns.

- Lesser Extent of Benchmarking: At present, there is only little work that has actually being benchmarked. Moreover, more number of the research work is found without comparative assessment this causes unreliability factor for implying the presented system in real-time environment or changing to some other environment or complexity.

\section{CONCLUSION}

This paper has discussed about the significance of techniques used for screening breast cancer. There are two part of this where one part is related to real-time practices of radiologist in hospitals and another is where researchers practices with breast phantoms. After more than a decade of investigation towards $\mathrm{CAD}$ based techniques, it was found that majority of the existing techniques are shrouded by pitfalls which requires serious attention. Moreover, in existing system more emphasis is laid on to the process of capturing the disease and less on improving the accuracy. Moreover studies towards classification are quite less to find as none of the studies till date are found to be benchmark. Hence, our future direction of study will be to introduce a modeling of CAD tool for diagnosing breast cancer effectively with higher accuracy. As there are lesser journals towards considering MRI image, our future experiments will be on the basis of MRI breast image to perform more critical detailing of tumors or lumps for reliable diagnosis.

\section{REFERENCES}

[1] B. G. Silverman, "Intelligent Paradigms for Healthcare Enterprises: Systems Thinking", Springer Science \& Business Media, pp. 266, 2005

[2] J.R. Benson, G.P.H. Gui, T.Tuttle, "Early Breast Cancer: From Screening to Multidisciplinary Management, Third Edition", CRC Press Medical, pp. 584, 2013

[3] T. Ayer, M.U.Ayvaci, Z. X.Liu, O.Alagoz, and E.S.Burnside, "Computer-aided diagnostic models in breast cancer screening",Imaging in medicine, Vol. 2, No. 3, pp.313-323,2010

[4] D.D.Feng, "Biomedical Information Technology", Academic Press, Technoogy \& Engineering, pp. 552, 2011

[5] A. Shukla, "Intelligent Medical Technologies and Biomedical Engineering: Tools and Applications: Tools and Applications", Idea Group Inc (IGI)-Business \& Economics, pp. 376, 2010

[6] B.K.Panigrahi, S.Das, P.N. Suganthan, S.S.Dash, "Swarm, Evolutionary, and Memetic Computing: First International Conference on Swarm, Evolutionary, and Memetic Computing, SEMCCO 2010, Chennai, India, December 16-18, 2010, Proceedings", Springer Computers, pp. 755, 2010

[7] C. Arya and R. Tiwari, "Expert system for breast cancer diagnosis: A survey," 2016 International Conference on Computer Communication and Informatics (ICCCI), Coimbatore, 2016, pp. 1-9.

[8] P. Darshini Velusamy and P. Karandharaj, "Medical image processing schemes for cancer detection: A survey," Green Computing Communication and Electrical Engineering (ICGCCEE), 2014 International Conference, Coimbatore, 2014, pp. 1-6.

[9] M. S. Islam, N. Kaabouch and W. C. Hu, "A survey of medical imaging techniques used for breast cancer detection," Electro/Information Technology (EIT), 2013 IEEE International Conference on, Rapid City, SD, 2013, pp. 1-5

[10] F.P. O'Malley, S.E. Pinder, A.M.Mulligan, "Breast Pathology: A Volume in the Series: Foundations in Diagnostic Pathology”, Elsevier Health Sciences, pp. 400, 2011

[11] Q. Li, R.M. Nishikawa, "Computer-Aided Detection and Diagnosis in Medical Imaging", Taylor \& Francis, pp. 454, 2015

[12] N.B.Hamad, K.Taouil, and M.S.Bouhlel, "Mammographic microcalcifications detection using discrete wavelet transform", International Journal of Computer Applications, Vol. 64, No. 21, 2013

[13] A. Sadana, N. Sadana, "Fractal Analysis of the Binding and Dissociation Kinetics for Different Analytes on Biosensor Surfaces",Elsevier, pp. 327, 2007

[14] R. Azmi, "A new markov random field segmentation method for breast lesion segmentation in MR images", Journal of medical signals and sensors, Vol.1, No. 3, 2011

[15] M.P. Sampat, M.K. Markey, \& A.C. Bovik, "Computer-aided detection and diagnosis in mammography", Handbook of image and video processing,Vol.2(1), pp.1195-1217, 2005

[16] R.N. Strickland, "Image-Processing Techniques for Tumor Detection", CRC Press, Technology \& Engineering, pp. 384, 2002

[17] S.Banik, R.M. Rangayyan, "Computer-Aided Detection of Architectural Distortion in Prior Mammograms of Interval Cancer", Morgan \& Claypool Publishers, pp. 169, 2013

[18] P.C. Walter, "Managing Cancer: Managing to Stay Alive", Palkon Publishing Services, pp. 384, 2004

[19] R.A. Castellino, "Computer aided detection (CAD): an overview", Cancer Imaging,Vol.5, No. 1, pp. 17, 2005

[20] J.S. Suri, R.M. Rangayyan, "Recent Advances in Breast Imaging, Mammography, and Computer-aided Diagnosis of Breast Cancer", Society of Photo Optical, pp. 972, 2006

[21] S. P. Singh, S. Urooj and A. Lay-Ekuakille, "Breast Cancer Detection Using PCPCET and ADEWNN: A Geometric Invariant Approach to Medical X-Ray Image Sensors," in IEEE Sensors Journal, vol. 16, no. 12, pp. 4847-4855, June15, 2016. 
[22] Q. Li, L. Wang, H. Song, H. Kono, P. Liu, H. Lu and T. Kikkawa, "Direct Extraction of Tumor Response Based on Ensemble Empirical Mode Decomposition for Image Reconstruction of Early Breast Cancer Detection by UWB," in IEEE Transactions on Biomedical Circuits and Systems, vol. 9, no. 5, pp. 710-724, Oct. 2015.

[23] A. Santorelli, E. Porter, E. Kang, T. Piske, M. Popović and J. D. Schwartz, "A Time-Domain Microwave System for Breast Cancer Detection Using a Flexible Circuit Board," in IEEE Transactions on Instrumentation and Measurement, vol. 64, no. 11, pp. 2986-2994, Nov. 2015

[24] X. Wang, T. Qin, R. S. Witte and H. Xin, "Computational Feasibility Study of Contrast-Enhanced Thermoacoustic Imaging for Breast Cancer Detection Using Realistic Numerical Breast Phantoms," in IEEE Transactions on Microwave Theory and Techniques, vol. 63, no. 5, pp. 1489-1501, May 2015.

[25] T. Yin, F. H. Ali and C. C. Reyes-Aldasoro, "A Robust and Artifact Resistant Algorithm of Ultrawideband Imaging System for Breast Cancer Detection," in IEEE Transactions on Biomedical Engineering, vol. 62, no. 6, pp. 1514-1525, June 2015.

[26] H. Song, H. Kono, Y. Seo, A. Azhari, J. Somei, E. Suematsu, Y. Watarai, T. OTA, H. Watanabe, "A Radar-Based Breast Cancer Detection System Using CMOS Integrated Circuits," in IEEE Access, vol. 3, no. , pp. 2111-2121, 2015.

[27] S. Kwon, H. Lee and S. Lee, "Image enhancement with Gaussian filtering in time-domain microwave imaging system for breast cancer detection," in Electronics Letters, vol. 52, no. 5, pp. 342-344, 332016.

[28] M. Jalilvand, X. Li, L. Zwirello and T. Zwick, "Ultra wideband compact near-field imaging system for breast cancer detection," in IET Microwaves, Antennas \& Propagation, vol. 9, no. 10, pp. 1009-1014, 7 162015.

[29] E. Kirshin, B. Oreshkin, G. K. Zhu, M. Popovic and M. Coates, "Microwave Radar and Microwave-Induced Thermoacoustics: DualModality Approach for Breast Cancer Detection," in IEEE Transactions on Biomedical Engineering, vol. 60, no. 2, pp. 354-360, Feb. 2013.

[30] A. Shahzad, M. O'Halloran, E. Jones and M. Glavin, "Prefiltered Beamforming for Early-Stage Breast Cancer Detection," in IEEE Antennas and Wireless Propagation Letters, vol. 12, no. , pp. 500-503, 2013.

[31] M. D. Hossain, A. S. Mohan and M. J. Abedin, "Beamspace TimeReversal Microwave Imaging for Breast Cancer Detection," in IEEE Antennas and Wireless Propagation Letters, vol. 12, no., pp. 241-244, 2013.

[32] M. Bassi, M. Caruso, M. S. Khan, A. Bevilacqua, A. D. Capobianco and A. Neviani, "An Integrated Microwave Imaging Radar With Planar Antennas for Breast Cancer Detection," in IEEE Transactions on Microwave Theory and Techniques, vol. 61, no. 5, pp. 2108-2118, May 2013.

[33] B. St. Peter, S. Yngvesson, P. Siqueira, "Development and Testing of a Single Frequency Terahertz Imaging System for Breast Cancer Detection," in IEEE Journal of Biomedical and Health Informatics, vol. 17, no. 4, pp. 785-797, July 2013

[34] G. Ruvio, R. Solimene, A. Cuccaro and M. J. Ammann, "Comparison of Noncoherent Linear Breast Cancer Detection Algorithms Applied to a 2D Numerical Model," in IEEE Antennas and Wireless Propagation Letters, vol. 12, no. , pp. 853-856, 2013.

[35] M. Guardiola, S. Capdevila, J. Romeu and L. Jofre, "3-D Microwave Magnitude Combined Tomography for Breast Cancer Detection Using Realistic Breast Models," in IEEE Antennas and Wireless Propagation Letters, vol. 11, no. , pp. 1622-1625, 2012.

[36] T. M. Grzegorczyk, P. M. Meaney, P. A. Kaufman, R. M. diFlorioAlexander and K. D. Paulsen, "Fast 3-D Tomographic Microwave Imaging for Breast Cancer Detection," in IEEE Transactions on Medical Imaging, vol. 31, no. 8, pp. 1584-1592, Aug. 2012.

[37] X. Wang, D. R. Bauer, R. Witte and H. Xin, "Microwave-Induced Thermoacoustic Imaging Model for Potential Breast Cancer Detection," in IEEE Transactions on Biomedical Engineering, vol. 59, no. 10, pp. 2782-2791, Oct. 2012.

[38] G. Angiulli, T. Isemie and S. Tringali, "Modeling Realistic Contrast Maps from MRI [EM Programmer's Notebook] Images for Microwave Breast Cancer Detection," in IEEE Antennas and Propagation Magazine, vol. 53, no. 1, pp. 113-122, Feb. 2011.

[39] A. J. Bekker, M. Shalhon, H. Greenspan and J. Goldberger, "Multi-View Probabilistic Classification of Breast Microcalcifications," in IEEE Transactions on Medical Imaging, vol. 35, no. 2, pp. 645-653, Feb. 2016.

[40] Z. Chen, H. Strange, A. Oliver, E. R. E. Denton, C. Boggis and R. Zwiggelaar, "Topological Modeling and Classification of Mammographic Microcalcification Clusters," in IEEE Transactions on Biomedical Engineering, vol. 62, no. 4, pp. 1203-1214, April 2015.

[41] M. J. Gangeh, H. Tadayyon, L. Sannachi, A. Sadeghi-Naini, W. T. Tran and G. J. Czarnota, "Computer Aided Theragnosis Using Quantitative Ultrasound Spectroscopy and Maximum Mean Discrepancy in Locally Advanced Breast Cancer," in IEEE Transactions on Medical Imaging, vol. 35, no. 3, pp. 778-790, March 2016.

[42] F. A. Spanhol, L. S. Oliveira, C. Petitjean and L. Heutte, "A Dataset for Breast Cancer Histopathological Image Classification," in IEEE Transactions on Biomedical Engineering, vol. 63, no. 7, pp. 1455-1462, July 2016.

[43] T. H. Vu, H. S. Mousavi, V. Monga, G. Rao and U. K. A. Rao, "Histopathological Image Classification Using Discriminative FeatureOriented Dictionary Learning," in IEEE Transactions on Medical Imaging, vol. 35, no. 3, pp. 738-751, March 2016.

[44] N. Uniyal, H. Eskandari, P. Abolmaesumi, S. Sojoudi, P. Gordon, L. Warren, R.N. Rohling, S.E. Salcudean, and M. Moradi, "Ultrasound RF Time Series for Classification of Breast Lesions," in IEEE Transactions on Medical Imaging, vol. 34, no. 2, pp. 652-661, Feb. 2015.

[45] F. Soares, F. Janela, M. Pereira, J. Seabra and M. M. Freire, "Classification of Breast Masses on Contrast-Enhanced Magnetic Resonance Images Through Log Detrended Fluctuation Cumulant-Based Multifractal Analysis," in IEEE Systems Journal, vol. 8, no. 3, pp. 929938, Sept. 2014.

[46] B. Krawczyk and G. Schaefer, "Breast Thermogram Analysis Using Classifier Ensembles and Image Symmetry Features," in IEEE Systems Journal, vol. 8, no. 3, pp. 921-928, Sept. 2014.

[47] X. Liu and J. Tang, "Mass Classification in Mammograms Using Selected Geometry and Texture Features, and a New SVM-Based Feature Selection Method," in IEEE Systems Journal, vol. 8, no. 3, pp. 910-920, Sept. 2014.

[48] T. Amaral, S. J. McKenna, K. Robertson and A. Thompson, "Classification and Immunohistochemical Scoring of Breast Tissue Microarray Spots," in IEEE Transactions on Biomedical Engineering, vol. 60, no. 10, pp. 2806-2814, Oct. 2013.

[49] A. B. Ashraf, S. C. Gavenonis, D. Daye, C. Mies, M. A. Rosen and D. Kontos, "A Multichannel Markov Random Field Framework for Tumor Segmentation With an Application to Classification of Gene ExpressionBased Breast Cancer Recurrence Risk," in IEEE Transactions on Medical Imaging, vol. 32, no. 4, pp. 637-648, April 2013.

[50] P. Filipczuk, T. Fevens, A. Krzyżak and R. Monczak, "Computer-Aided Breast Cancer Diagnosis Based on the Analysis of Cytological Images of Fine Needle Biopsies," in IEEE Transactions on Medical Imaging, vol. 32, no. 12, pp. 2169-2178, Dec. 2013.

[51] E. E. Tripoliti, D. I. Fotiadis and G. Manis, "Automated Diagnosis of Diseases Based on Classification: Dynamic Determination of the Number of Trees in Random Forests Algorithm," in IEEE Transactions on Information Technology in Biomedicine, vol. 16, no. 4, pp. 615-622, July 2012.

[52] M. M. Dundar, S. Badve, G. Bilgin, V. Raykar, R. Jain, "Computerized classification of intraductal breast lesions using histopathological images," in IEEE Transactions on Biomedical Engineering, vol. 58, no. 7, pp. 1977-1984, July 2011. 
[53] M. Fraschini, "Mammographic masses classification: novel and simple signal analysis method," in Electronics Letters, vol. 47, no. 1, pp. 14-15, January 62011.

[54] A. Mencattini, M. Salmeri, G. Rabottino and S. Salicone, "Metrological Characterization of a CADx System for the Classification of Breast Masses in Mammograms," in IEEE Transactions on Instrumentation and Measurement, vol. 59, no. 11, pp. 2792-2799, Nov. 2010.

[55] J. Teo, Y. Chen, C.B. Soh, E. Gunawan, K. S. Low, T.C. Putti and S-C. Wang, "Breast Lesion Classification Using Ultrawideband Early Time Breast Lesion Response," in IEEE Transactions on Antennas and Propagation, vol. 58, no. 8, pp. 2604-2613, Aug. 2010.



AUTHOR PROFILE

Vidya $\mathbf{K}$ has obtained her B. E. degree in the year 1998 from Mysore University and M. Tech. degree in the year 2003 from VTU. The area of her research interest is image processing. Currently doing research in processing of MRI images.

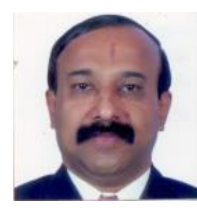

Dr. M Z Kurian has obtained his B. E. degree in the year 1982 and $M$. Tech. degree in the year 1988. He also awarded $\mathrm{Ph}$. D. in the year 2010. Presently he is working as Professor and Head of the Department of Electronics and Communication Engineering at Sri Siddhartha Institute of Technology, India. The areas of his research interest are image processing and software engineering. 